\title{
Searching for Variable Sources in the Canadian Galactic Plane Survey
}

\section{A. Del Rizzo ${ }^{* a}$, A. D. Gray ${ }^{a}$, S. M. Dougherty ${ }^{a}$ and A. R. Taylor ${ }^{b}$}

${ }^{a}$ National Research Council Canada, Herzberg Institute of Astrophysics, Dominion Radio Astrophysical Observatory PO Box 248, White Lake Rd. Penticton, BC, Canada V2A $6 \mathrm{~J} 9$

${ }^{b}$ Department of Physics and Astronomy, University of Calgary 2500 University Dr. NW, Calgary, AB, Canada T2N $1 N 4$

E-mail: dave.delrizzo@nrc-crnc.gc.ca

The Canadian Galactic Plane Survey (CGPS) radio continuum database is ideally suited to searches for radio variable sources, allowing a deep search for variability to be carried out on tens of thousands of compact radio sources in the Galactic Plane. As a consequence of the multiday observing method used in the CGPS, sources with variations on time scales of hours, days and months, can be identified directly from the data. In this talk, we present a method of identifying intra-day variations from these data, and illustrate its application on survey regions.

Bursts, Pulses and Flickering:Wide-field monitoring of the dynamic radio sky

June 12-15 2007

Kerastari, Tripolis, Greece

${ }^{*}$ Speaker. 


\section{Observations}

The Synthesis Telescope (ST) at the Dominion Radio Astrophysical Observatory is an aperture synthesis interferometer located near Penticton, British Columbia, Canada. The ST comprises seven 9-m diameter radio telescopes aligned in an east-west configuration. Three of the antennas are moveable along a section of rail $300 \mathrm{~m}$ in length, while the remainder are fixed in a configuration with a maximum baseline of $617.1 \mathrm{~m}$. The ST provides images with a resolution of 1 minute of arc across a field of view approximately 2.5 degrees in diameter at an observing frequency of $1420 \mathrm{MHz}$.

One 'observation' with the ST comprises 12 contiguous hours of data in a single array configuration. One 'field' comprises twelve 'observations', each in a different array configuration so that all possible baselines are measured. Reconfigurations are done approximately every 4 days, so a full synthesis of a given field takes place over the course of 7-8 weeks. Once observed, the raw data for all 'observations' are archived.

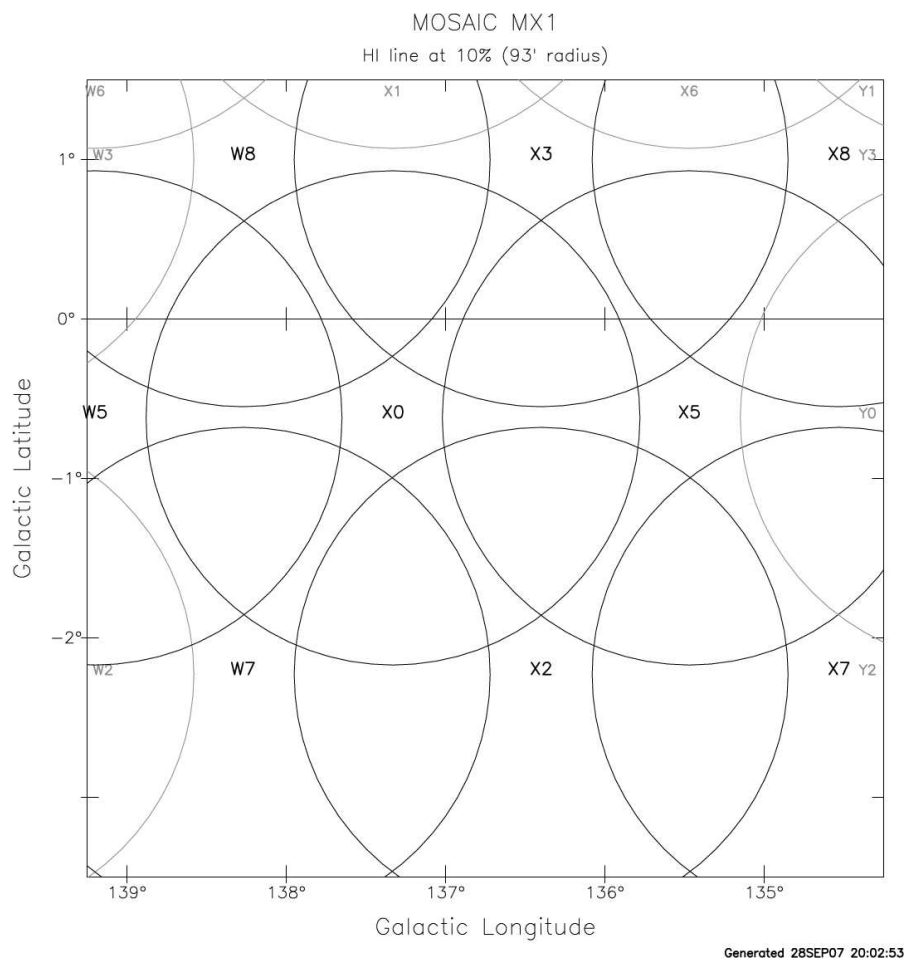

Figure 1: An example of the hexagonal grid spacing in a section of the Canadian Galactic Plane Survey. The circles represent the effective edges of ST fields at $1420 \mathrm{MHz}$.

Since 1995 the ST has been used primarily for observing fields for the Canadian Galactic Plane Survey, which is surveying the Galactic Plane from $l=195^{\circ}$ to $l=50^{\circ}$ in the latitude range from $b=-3^{\circ}$ to $b=+5^{\circ}$. The CGPS also includes a high latitude extension from $l=100^{\circ}$ to $l=114^{\circ}$ between $b=5^{\circ}$ and $b=17^{\circ}$. There are approximately 450 fields in the survey, observed on a hexagonal grid with spacing just over one half-power beamwidth, so nearly all of each field overlaps with one or more others. 
The survey was completed in three phases, and observations in each phase were scheduled for observing efficiency rather than contiguous coverage, so overlapping fields may have been observed days, months, or even years apart. The large spatial overlap and temporal span within and between adjacent fields allows us to obtain time-series data for numerous compact radio sources. The data that have been, and continue to be, collected for the CGPS thus provide a unique resource for studying source variability.

\section{Search Algorithm}

A simple algorithm has been developed in order to exploit the CGPS data archive. The aim of the algorithm is to perform an initial mining of the data, detecting any temporal variations in intensity of compact radio sources that occurred during the course of the CGPS observations.

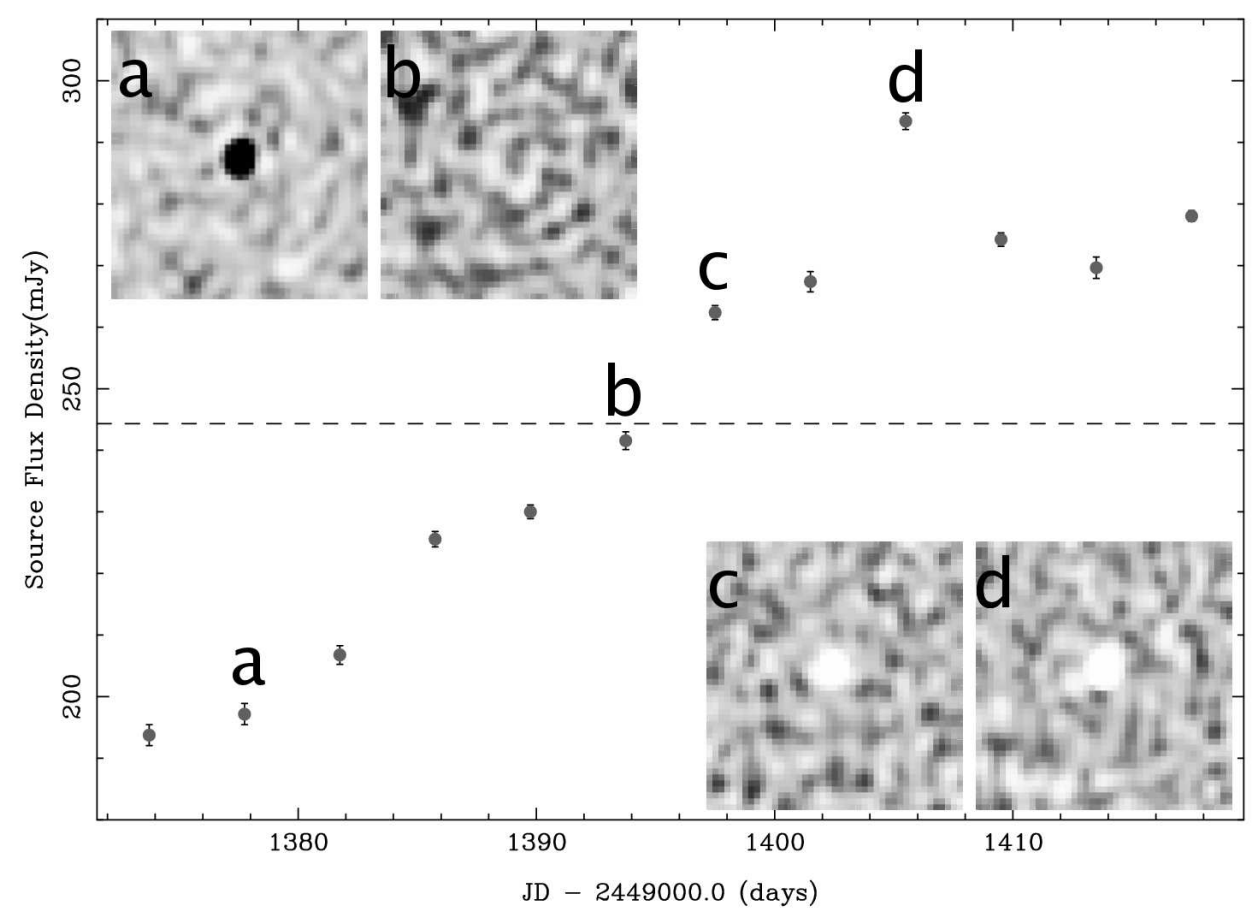

Figure 2: $1420 \mathrm{MHz}$ radio light curve of J0335+599. Insets $a$ through $d$ show a section of the difference images centred on the candidate source for the corresponding points on the radio light curve. The dashed line represents the flux density of the source in the reference image.

The algorithm can be described in a few brief steps:

1. An image of a single field is made and CLEANed, then convolved to the largest common spatial resolution of the constituent observations. This is the 'reference' image. 
2. Images of each observation are then created, CLEANed, and convolved in a similar manner to the reference image. These are the 'daily images'.

3. Differences between each daily image and the reference image are searched for peaks that rise more than 5 times the RMS of the difference image, and a 2-D Gaussian fit is performed in the corresponding daily image to obtain the position and flux density.

4. The list of all identified sources is filtered (see Section 2.1) to remove false detections.

5. A radio light curve derived from all overlapping fields is then extracted for those candidate sources remaining.

6. Light curves are inspected by eye and those sources that exhibit plausible variability are investigated further.

Analysis of an observed field is only performed upon completion of the full synthesis.

\subsection{Search Filters}

The list of candidate variable sources obtained from each field is subjected to software filters designed to assist in the elimination of false detections. These filters can be briefly described as follows:

- Variability Filter: the peak-to-peak variation of the flux density of a candidate must be larger than a chosen limit. For bright sources with an average flux density of more than $1 J y$, the peak-to-peak variability must be greater than $10 \%$ of the source flux measured in the reference image. For weaker sources a $15 \%$ threshold is used.

- Flux Error Filter: the formal error of the fitted flux density in the reference image must be less than $10 \%$ of the source flux density, to eliminate sources that are not well-fitted by the algorithm (e.g., not compact, or multiple sources in the fitting box).

- Point Scatter Filter: the standard deviation of all daily flux density measurements for a candidate must be greater than $5 \%$ of the fitted flux density in the reference image.

- Noise Filter: The standard deviation of all daily flux density measurements for a candidate must exceed the RMS noise in the daily images. This filter ensures that any detections are indeed above the noise level in a daily observation.

The listed filters have been tuned according to instrument parameters and results from iterative data analysis. Refinement of these threshold parameters is ongoing.

\section{Preliminary Results and Examples}

Applying this algorithm to over 300 fields to date has yielded approximately 900 candidate sources, or 3 sources per field on average. As a verification of the algorithm, known variable radio sources lying in the survey region were examined. Some of these results are presented in figures 3 through 5 . 


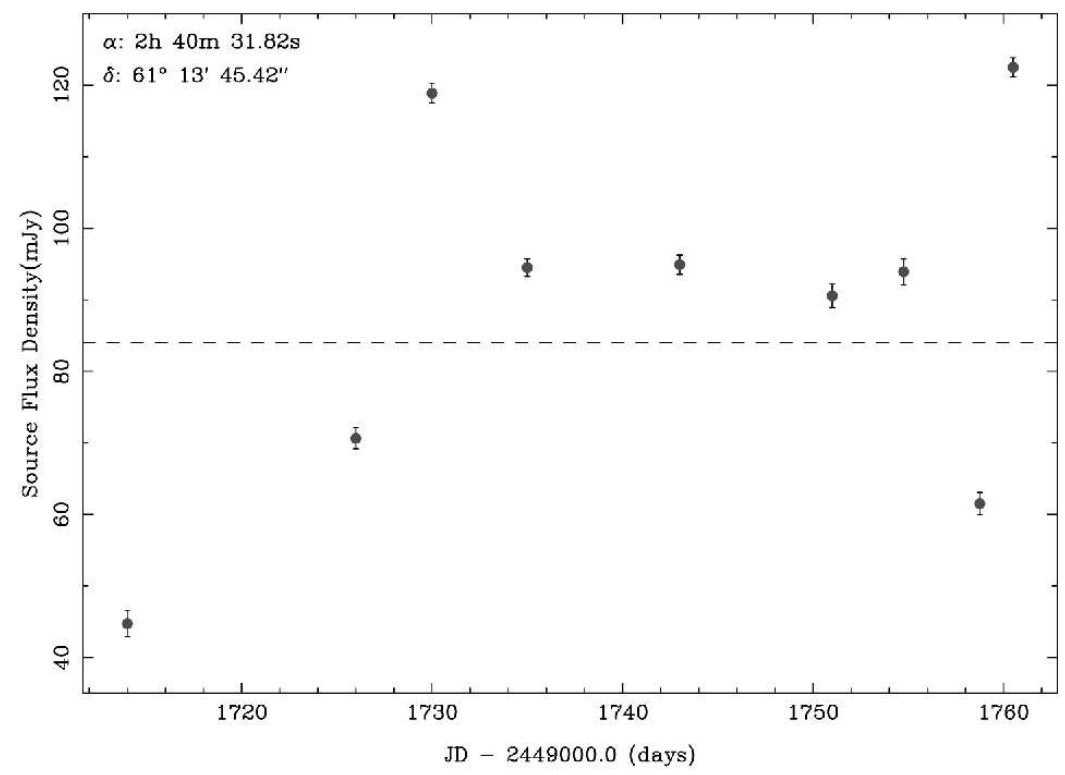

Figure 3: Radio light cuve of LSI $+61^{\circ} 303$ at $1420 \mathrm{MHz}$. This source is an unusual high-mass X-ray binary, known to undergo non-thermal radio outbursts every 26.5 days (Taylor \& Gregory). The dashed line indicates the flux density of the source measured in the reference image.

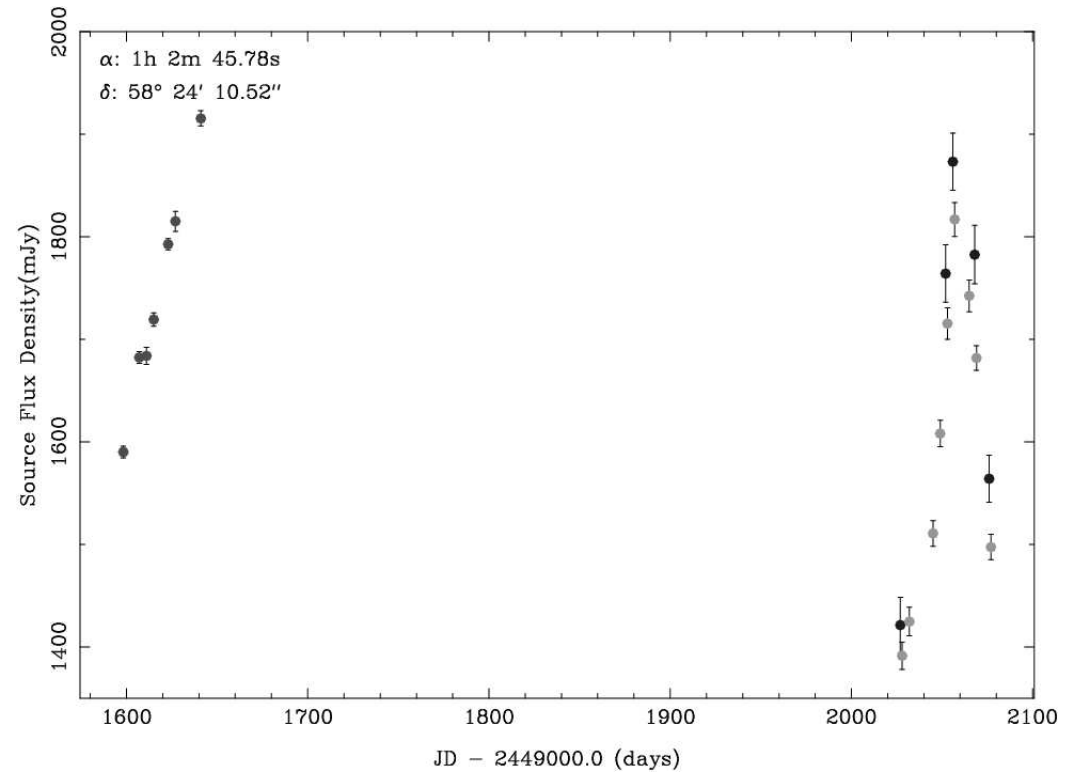

Figure 4: $1420 \mathrm{MHz}$ radio light curve of pulsar J0102+58 obtained from the analysis of three separate fields. The first observation took place in May 1997, followed by two concurrent observations in August 1998. Differences in flux density error between the two epochs are attributed to source position relative to the centre of the observed field.

\section{Future Work}

As experience is gained with applying this algorithm to the CGPS data set, parameters will 


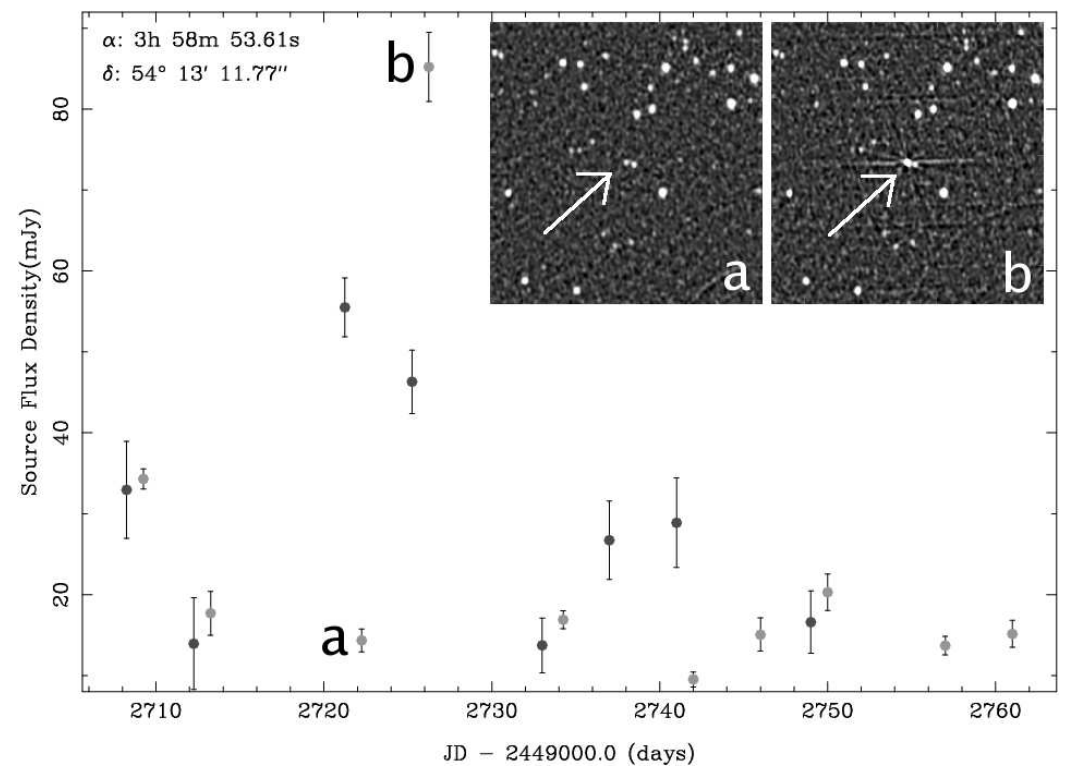

Figure 5: $1420 \mathrm{MHz}$ radio light curve of pulsar J0358+5413 obtained from the analysis of two concurrently observed fields. Insets $a$ and $b$ show the source during both quiescent $(a)$ and bursting $(b)$ phases of the light curve. The spoke-like structures seen radiating from the source in inset $b$ are evidence of intra-day variability.

be tuned to optimize the efficiency of identification of variable candidates and minimize both false positives and false negatives. The goal is to apply the algorithm to all 450 CGPS fields plus additional regions where there are multiple overlapping fields observed for other projects (150 fields). Ultimately, a classification scheme will be developed and a catalogue of variable sources produced.

\section{Acknowledgements}

The Canadian Galactic Plane Survey (CGPS) is a Canadian project with international partners. The Dominion Radio Astrophysical Observatory is operated as a national facility by National Research Council Canada. The CGPS is supported by the Natural Sciences and Engineering Research Council of Canada.

\section{References}

[1] J.A. Högbom, Aperture Synthesis with a Non-Regular Distribution of Interferometer Baselines, 1974, $A \& A S, 15,417-426$.

[2] T.L. Landecker, et al., The synthesis telescope at the Dominion Radio Astrophysical Observatory, 2000, A\&AS, 145, 509-524.

[3] A.R. Taylor, et al., The Canadian Galactic Plane Survey, 2003, AJ, 125, 3145-3164.

[4] A.R. Taylor and P.C. Gregory, Periodic Radio Emission from LSI +61 ${ }^{\circ} 303,1982$, ApJ, 255, 210-216. 